\section{Cushing syndrome secondary to late onset adrenal hyperplasia: presentation and challenges of management}

\author{
Muzzammil Abdullahi, ${ }^{1}$ \\ Mamuda Atiku, ${ }^{2}$ \\ Imam Mohammed Ibrahim ${ }^{3}$ \\ Departments of ${ }^{1}$ Surgery, ${ }^{2}$ Anaesthesia, \\ ${ }^{3}$ Histopathology, Bayero University, \\ Aminu Kano Teaching Hospital, Kano, \\ Nigeria
}

\begin{abstract}
Cushing's syndrome is a clinical disorder caused by overproduction of cortisol. Adrenal adenoma is the cause in $5 \%$ of cases of Cushing syndrome. ACTH-independent Cushing's syndrome in $90 \%$ is caused by unilateral adrenal tumors. Of these, adenomas are the cause in $80 \%$ of the cases, while the others are adrenocortical carcinoma. Rare causes of Cushing's syndrome include adrenal hyperplasia. Overproduction of cortisol results in weakened protein structures leading to protuberant abdomen and poor wound healing, glucose is converted to fat and deposited in the abdomen, supraclavicular fossa and cheeks. Other presentations are diabetes, hypertension, osteoporosis, fractures, impaired immune function, glucose intolerance, and psychosis. We report a 21-year-old female who presented with clinical symptoms of newly diagnosed hypertension and diabetes mellitus which after poor response to treatment she was evaluated and diagnosed to have Cushing syndrome. She subsequently had left adrenalectomy and did very well with resolution of patients' symptoms. The histology came out to be diffuse adrenal hyperplasia.
\end{abstract}

\section{Introduction}

Cushing's syndrome is a clinical disorder caused by overproduction of cortisol. In endogenous cases most $(\geq 80 \%)$ are due to bilateral adrenocortical hyperplasia stimulated by overproduction of pituitary adrenocorticotropic hormone (corticotrophin, ACTH), known as Cushing's disease. About $10 \%$ of cases are due to the ectopic production of ACTH from non-pituitary tumors. Adrenal adenoma is the cause in $5 \%$ of cases and carcinoma in 5\%. ${ }^{1}$ ACTH-independent Cushing's syndrome in $90 \%$ is caused by unilateral adrenal tumors. Of these, adenomas are the cause in $80 \%$ of the cases, while the others are adrenocortical carcinoma. $^{2}$ Rare adrenal causes of Cushing's syndrome include macronodular adrenal hyperplasia, diffuse adrenal hyperplasia, primary pigmented nodular adrenal disease (sporadic or as part of Carney's complex) and McCune-Albright syndrome ${ }^{2}$ and topical or nasal steroid administration. ${ }^{3}$

The cells in adrenal hyperplasia resemble those of the zona fasciculata of the normal adrenal cortex. Overproduction of cortisol leads to catabolism with liberation of amino acids from muscles tissue, which is transformed into glucose and glycogen in the liver. ${ }^{1,4,5}$ The resulting weakened protein structures cause a protuberant abdomen and poor wound healing, glucose is converted to fat and deposited in the abdomen, supraclavicular fossa and cheeks. Diabetes and Hypertension are present in 20\% and $90 \%$ of cases respectively. ${ }^{1}$ Others are osteoporosis, fractures, impaired immune function, glucose intolerance, and psychosis. ${ }^{4}$ In its overt expression Cushing's syndrome is usually easily recognized but subclinical forms can represent a significant challenge for physicians, frequently missed in the diagnostic workup.Here we present a clinical case of a young woman whose presentation pose initial challenge to diagnosis of Cushing syndrome in a resource poor center repeatedly evaluated for common medical problems; it was later diagnosed with the demonstration of a unilateral adrenal tumor, which was successfully removed with resolutions of symptoms.

\section{Case Report}

A 21-year-old university undergraduate student presented to our hospital with a 3 months history of progressive body swelling, weight gain, headache and skin lightening. No past medical history of note except for a breast lump excision she had about 6 months prior to onset of these symptoms which the histology confirmed to be fibrocystic breast disease. Around the same time, she was diagnosed to have hypertension and diabetes mellitus. She gained weight of about $23 \mathrm{~kg}$ (from $62 \mathrm{~kg}$ to $85 \mathrm{~kg}$ ) over 9 months with the obesity mainly around the trunk and the face. She was initially been managed for the aforementioned diseases however despite being on high doses of antihypertensive (Amlodipine and losartan) and subcutaneous insulin the high blood pressure and blood sugar remained largely uncontrolled. She was then evaluated for possible Cushing syndrome; the following results were obtained: Serum Cortisol, $50 \mu \mathrm{g} / \mathrm{dL}$ (NR 5-23 $\mu \mathrm{g} / \mathrm{dL}$ in the morning and $3-13 \mu \mathrm{g} / \mathrm{dL}$ in the after-
Correspondence: Muzzammil Abdullahi, Department of Surgery, Aminu Kano Teaching Hospital, Kano, Nigeria.

Tel.: +080.35678205 .

E-mail: muzzammilabdullahi@yahoo.co.uk

Key words: Cushing syndrome; adrenal hyperplasia; presentation; management.

Contributions: the authors contributed equally

Conflict of interest: the authors declare no potential conflict of interest.

Funding: none.

Received for publication: 29 July 2018.

Revision received: 24 September 2018.

Accepted for publication: 24 September 2018.

This work is licensed under a Creative Commons Attribution NonCommercial 4.0 License (CC BY-NC 4.0).

(C) Copyright M. Abdullahi et al., 2018

Licensee PAGEPress, Italy

Pyramid Journal of Medicine 2018; 1:8 doi:10.4081/pjm.2018.8

noon); Plasma adreno corticotrophin hormone (ACTH), below $1 \mathrm{pg} / \mathrm{mL}$ (NR 7.2 to $63.3 \mathrm{pg} / \mathrm{mL}) ;$ Serum aldosterone, 23.8 ng/dL (NR 2.52-39 ng/dL upright and 1.76$23.2 \mathrm{ng} / \mathrm{dL}$ supine); Urinary vanillylmandelic acid, $5.37 \mathrm{mg}$ /day (NR up to 15 $\mathrm{mg} /$ day).

The investigations show a high serum cortisol level, low plasma ACTH level and normal serum aldosterone and urinary VMA levels. The abdominal CT Scan showed a left adrenal tumor which was measuring $24.4 \times 22.3 \mathrm{~mm}$ (Figure 1), however, the brain CT scan was essentially normal (Figure 2). Overall features were those of Cushing syndrome secondary to Adrenal tumor. Other investigations including electrocardiogram, echocardiogram, renal function tests and complete blood count were within normal limits. Symptoms were worsening as evidenced by continued weight gain, severe headache, blurring of vision and increasing appetite despite being on treatment with antihypertensive, insulin and ketaconazole. Hence the decision to do adrenalectomy for her. She had open adrenalectomy via flank $11^{\text {th }}$ rib incision under general anaesthesia (balanced anaesthesia). Both surgery and anaesthesia were uneventful except for fluctuation of the blood pressure and arrhythmia which were managed appropriately. A well circumscribed globular mass measuring $4 \times 3 \times 2 \mathrm{~cm}$ was found on the upper pole of the left kidney and was excised. The mass is shown in Figure 3. The ipsilateral kidney 


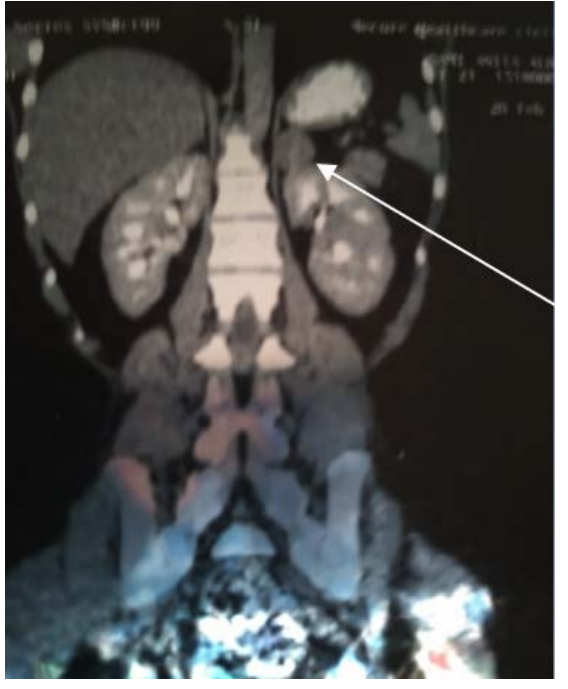

Figure 1. Showing left adrenal tumor (24.4 $\times 22.3 \mathrm{~mm}$ ).

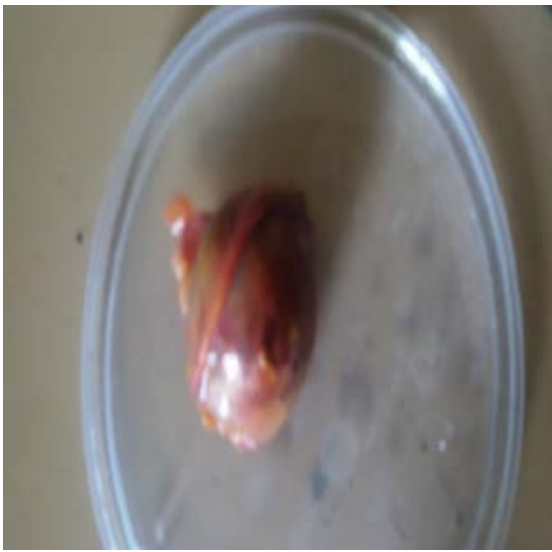

Figure 3. Left adrenal tumor.

was normal. The histology showed a diffuse adrenal hyperplasia (Figure 4).

In the post-operative period the blood pressure remains within normal limit (between $110 / 70 \mathrm{mmhg}$ to $130 / 90 \mathrm{mmhg}$ ), however, the blood sugar remained high hence she was continued on subcutaneous insulin. She was also commenced on intravenous hydrocortisone starting with a dose of $200 \mathrm{mg}$.

She remained stable afterward, the blood pressure remained normal, the weight gradually decreased, and the high blood glucose was successfully being controlled. At 3 months post operatively, she recorded a significant weight loss to $78 \mathrm{~kg}$ (a decrease of $7 \mathrm{~kg}$ ), the blood glucose was normal $5.5 \mathrm{mmol} / \mathrm{L}$, the blood pressure and the serum cortisol became $8 \mu \mathrm{g} / \mathrm{dL}$. The

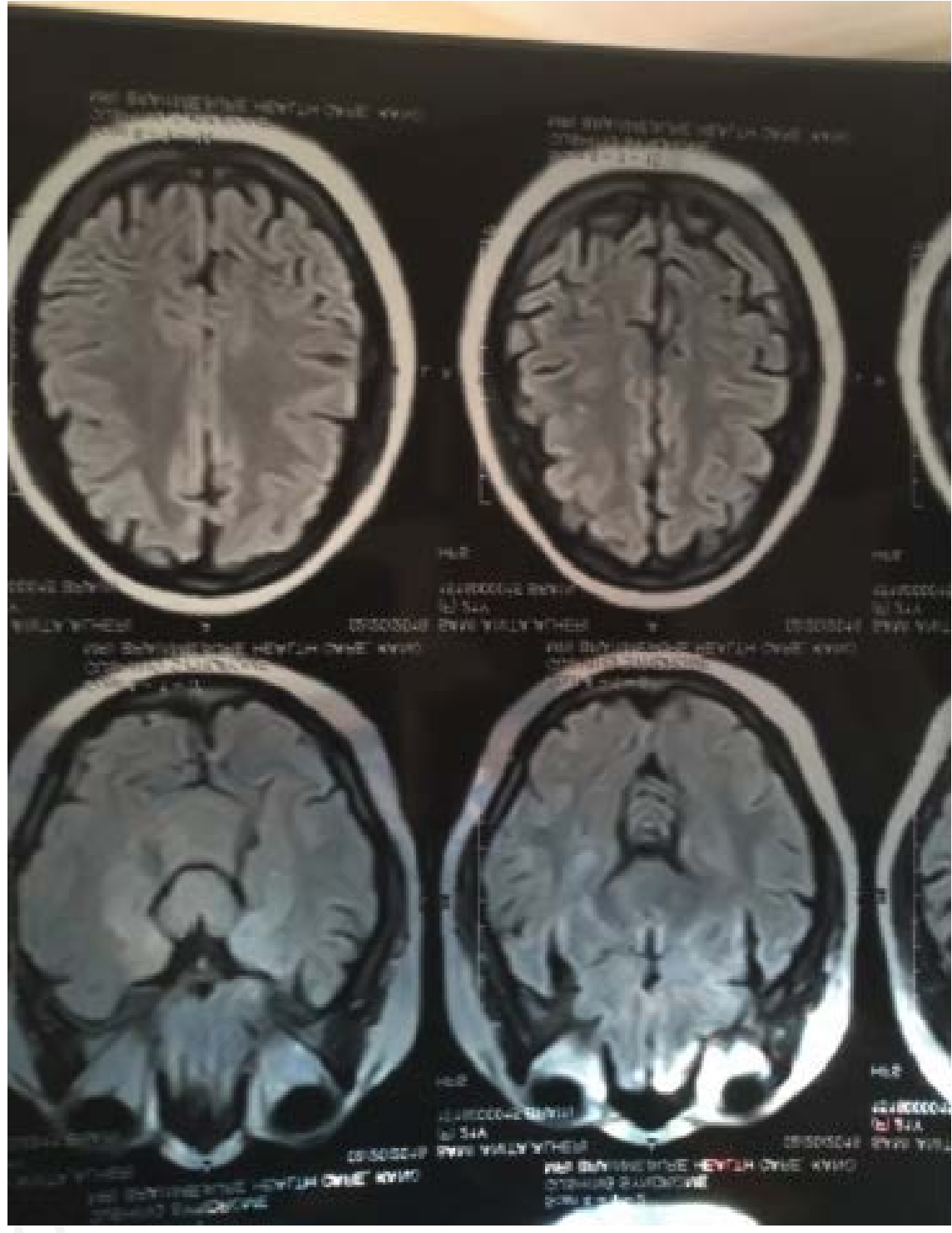

Figure 2. Normal brain computed tomography scan.
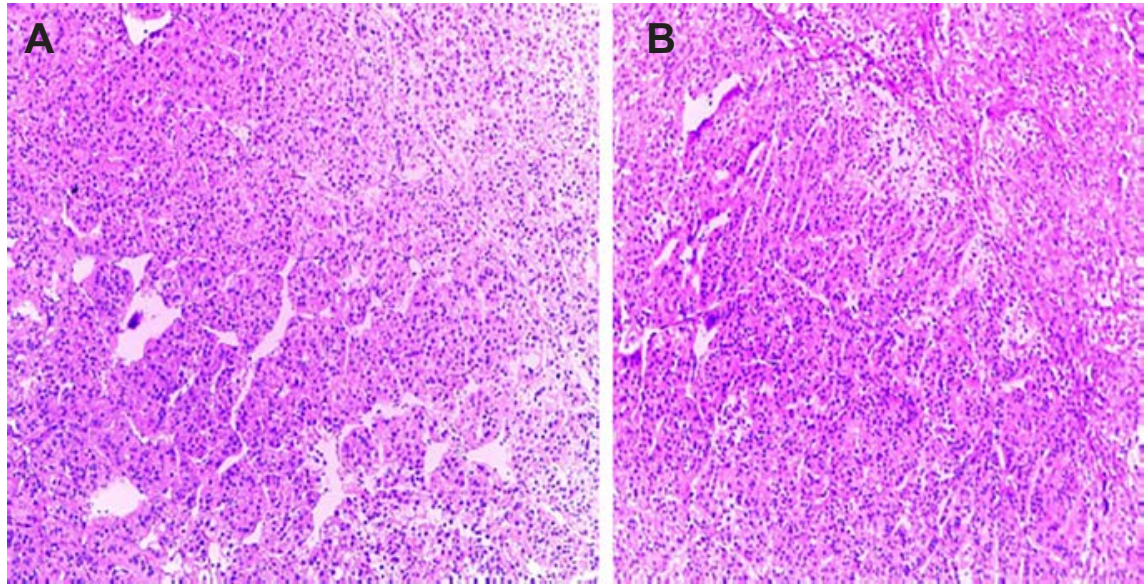

Figure 4. Diffuse adrenal hyperplasia showing sheets and nests of uniformly size cells with small, round nuclei and abundant granular cytoplasm disposed in Zellbellen pattern. Areas of lipomatous metaplasia are also seen $(H \& E$ 100 $\times)$. 
cortisone therapy was also gradually scaled down to zero which patient tolerated well.

\section{Discussion}

Cushing syndrome is a rare disease worldwide. However, its exact incidence is probably underestimated due to wide spectrum of presentation from subclinical to severe symptoms. In a European population-based study, the annual incidence of endogenous Cushing syndrome is 0.6 per million per year (adrenal adenoma), and 0.2 per million per year (adrenal carcinoma). ${ }^{2}$

The female-to-male incidence ratio is approximately 5:1 for Cushing syndrome due to an adrenal or pituitary tumor with peak incidence around the age of 25-40 years. $^{2}$

Adrenal hyperplasia is one of the causes of Cushing syndrome that is often regarded as extremely rare but which could be that because it's overlooked and this explains the few reports of the disease from around the globe and none of such from our environment. ${ }^{6}$

The index patient presented with an initially symptoms of predominantly common medical conditions and later other classical symptoms of Cushing syndrome. This and couple with rarity of the disease led to an initially difficult clinical diagnosis of the condition. Also, the initial routine investigations of the patient including an abdominal sonogram missed the diagnosis. Abdominal sonogram as reported in other studies is very poor in diagnosing an adrenal mass. ${ }^{7}$ Hence patient was being managed for other diseases such as hypertension and diabetes for months before eventually diagnosing the primary disease. This was also the case in some other reports on Cushing syndrome as reported by Denegeri A from Italy. ${ }^{8}$ The non- availability of investigations facilities for serum cortisol and a CT scan in the first managing hospital also further delayed the diagnosis.

\section{Conclusions}

The high index of suspicion of rare diseases such as Cushing syndrome in patients with presentations of mostly common diseases such as hypertension, diabetes and obesity couple with early referral in suspicious diagnosis will limit cases of missed and delayed diagnosis with its attendant fatality. Also availability and use of screening tools for serum hormonal tests such as cortisol and CT Scans will aid in early and accurate diagnosis of adrenal masses and diseases associate with it.

\section{References}

1. Christopher JK. Disorders of the Adrenal Glands. In: Tanagho EA,
McAninch JA. Smith's general urology. 17th ed. New York: MC Graw Hill; 2008. pp 490-506.

2. Susmeeta TS, Lynnette KN, Richard AF. Cushing's syndrome: epidemiology and developments in disease management. Clin Epidemiol 2015;7:281-93.

3. Isaac OO, Abiola O, Oduwole EO, et al. Iatrogenic Cushing's syndrome in children following nasal steroid. Pan Afr Med J 2014; 17:237.

4. Ha Cam TN, Romesh K. Endogenous Cushing Syndrome. 2017. Available from: https://emedicine. medscape.com/article/2233083overview\#showall2017.

5. Constantine AS. Cushing syndrome caused by adrenocortical tumors and hyperplasias (corticotrophin-independent Cushing syndrome. Endocr Dev 2008;13:117-32.

6. Kotb AF, Atta MA. Unilateral nodular adrenal hyperplasia: Case series. Afr J Urol 2016;175-7.

7. Nicolaus AW, Ali B, Mouhammed AH, et al. Cushing Syndrome: Diagnostic Workup and Imaging Features, With Clinical and Pathologic Correlation. Am J Roentgenol 2017;209:19-32.

8. Denegri A, Artom N, Moretti S, et al. A Long-Standing Subtle Cushing's syndrome Induced by a Unilateral Macronodular Adrenal Hyperplasia J Anesth Clin Res. 2014;6:494. 